\title{
Linx
}

Revue des linguistes de l'université Paris X Nanterre

$55 \mid 2006$

Thème et thématisation

\section{Ordre des mots et thématisation en latin}

\section{Bernard Bortolussi}

\section{OpenEdition}

\section{Journals}

Édition électronique

URL : http://journals.openedition.org/linx/378

DOI : $10.4000 /$ linx.378

ISSN : 2118-9692

\section{Éditeur}

Presses universitaires de Paris Nanterre

\section{Édition imprimée}

Date de publication : 1 décembre 2006

Pagination : $33-47$

ISSN : 0246-8743

\section{Référence électronique}

Bernard Bortolussi, «Ordre des mots et thématisation en latin », Linx [En ligne], 55 | 2006, mis en ligne

le 21 février 2011, consulté le 30 avril 2019. URL : http://journals.openedition.org/linx/378 ; DOI :

10.4000/linx.378 


\title{
Ordre des mots et thématisation en latin
}

\author{
Bernard Bortolussi \\ ArScAn, Paris $X$ \\ bernard.bortolussi@u-paris10.fr
}

\section{Introduction}

La description des procédés de Thématisation dans une langue morte se heurte à une difficulté majeure : la réalisation phonologique des énoncés particuliers nous échappe presque complètement; ni les pauses, ni les courbes intonatives ne nous sont directement accessibles. Pour contourner cette difficulté, nous devons d'abord faire l'hypothèse que le latin connait effectivement des procédés de Thématisation. Il s'agit de recourir ensuite à des critères autres qu'intonatifs.

Les études qui ont été menées, notamment dans le cadre des Grammaires Fonctionalistes, ont permis d'identifier un certain nombre de procédés de mise en relief du Thème (ou Topique) :

- par antéposition sous la forme d'un constituant prépositionnel (préposition de + ablatif $:$

(1a) de Tirone, cura, quaeso, quod facis, ut sciam quid is agat (Cic. Att. 10,4,12) « concernant Tiron, veille, je te prie, à continuer de m'informer de ce qu'il fait »

(1b) de Hispaniis non dubitabat quin Caesaris essent (Cic. Att. 10,4,8) « concernant les Espagnes, il ne doutait pas qu'elles ne fussent aux mains de César »

(1c) de multitudine quoniam quod satis esset admonui, de obscuritate pauca dicam (Varro LL. 6,40 ) «puisque à propos du nombre (des verbes) j’ai donné des informations suffisantes, à propos de leur obscurité je dirai peu de choses» 
Bernard Bortolussi

- par antéposition sous la forme d'une proposition :

(2a) Nam quod se similem esse Catilinae gloriari solet, scelere par est illi, industria inferior (Cic. Phil. 4,5)

«En effet, concernant son habituelle vantardise, comme quoi il ressemblerait à Catilina (litt. le fait qu'il a l'habitude de se vanter d'être semblable à Catilina), pour le crime, il le vaut, mais pour l'ardeur au travail, il est inférieur. »

(2b) quaeque ipsi non tribuunt amicis, haec ab iis desiderant (Cic. Lael. 82) « et cela même qu'ils ne donnent pas à leurs amis, ils le leur demandent »

- par antéposition d'un SN au nominatif (nominativus pendens) :

(3a) Ager rubricosus et terra pulla, materina, rudecta, harenosa, item quae aquosa non erit, ibi lupinum bonum fiet. (Cato agr. 34,2)

«Un sol rouge, une terre noire, matérine, mêlée de gravats, sablonneuse, de même une terre où viendra de l'eau, là le lupin poussera bien. »

(3b) baec tota fabella ueteris et plurimarum fabularum poetriae, quam est sine argumento, quam nullum inuenire exitum potest! (Cic. Cael. 64)

«cette scène, dans son ensemble, d'une poésie ancienne et caractéristique de quantité de pièces, combien elle est dépourvue d'intrigue, combien il est impossible de lui trouver une fin!»

- par antéposition du Thème dans la progression thématique, linéaire aussi bien qu'à Thème constant :

(4a) Erum eccum video buc Simonem ... incedere. Ex boc sepulcro uetere viginti minas / effodiam ego hodie (Plaut. Pseud. 410-413)

"Je vois qu'arrive ici mon maittre Simon. De ce vieux sépulcre je m'en vais aujourd'hui déterrer vingt mines »

(4b) Itaque se suaque omnia Caesari dediderunt. In quos eo grauius Caesar vindicandum statuit... (Caes. Gall. 3,16,3-4)

"C'est pourquoi ils se livrèrent corps et biens à César. Contre eux César décida de sévir avec d'autant plus de sévérité...»

L'objet de la présente étude sera d'examiner les aspects syntaxiques de la Thématisation. Je partirai de l'analyse de Touratier (1994), qui parle d'Extraposition pour tout constituant anticipé dans la chaîne syntagmatique, pour proposer des critères de différenciation entre plusieurs types d'Extraposition. Un second point concernera la Thématisation dans les propositions subordonnées. Enfin sera évoquée l'analyse syntaxique de la Thématisation dans le cadre des théories chomskyennes. 


\section{Plusieurs formes d'Extraposition à gauche en latin ?}

1.1. Chr. Touratier (1994, p. 187) fait l'analyse suivante de l'exemple (3a) : «Ce nominatif n'est pas, à proprement parler, hors syntaxe $(.$.$) ; il fait partie de$ l'organisation structurale de l'énoncé et remplit en fait une fonction syntaxique que nous appellerons extraposition, et qui a en commun avec le sujet d'une phrase minimale d'indiquer ce sur quoi le locuteur entend parler, c'est-à-dire le Thème ou le support informatif de l'énoncé. » Il étend cette analyse par extraposition à plusieurs sortes de constituants (1994, p. 709 sqq) :

a) les constituants sans fonction : il vise non seulement les constituants en de + abl. et le nominativus pendens, mais aussi les pronoms personnels au nominatif, notamment quand la forme verbale ne réclame pas de sujet :

(5) Tu uero, inquit, Antoni, perge ut instituisti (Cic. de orat. 2,124)

"Quant à toi, dit-il, Antoine, continue comme tu as commencé »

De manière générale les connecteurs autem ou uero (cf. aussi (6b)) sont caractéristiques de l'introduction du Thème.

b) les constituants qui n'occupent pas une place canonique dans l'énoncé ; les critères pour établir cette dérogation à l'ordre non-marqué sont entre autres :

- l'antéposition par rapport aux constituants définissant le type de phrase :

(6a) quid? Theophrastus mediocriterne delectat ... ? (Cic. fin. 1,6)

«Eh quoi ? Théophraste, est-ce qu'il plaît moyennement? »

(6b) Demosthenen uero ... quis est qui non sciat semper ante speculum ... causas meditatum ? (Apul., apol. 15,8)

«Quant à Démosthène, qui ignore qu'il a toujours répété ses plaidoyers devant un miroir?»

(6c) Familiae male ne sit, ne algeat, ne esuriat (Cato agr. 5,2)

"Concernant les esclaves, qu'ils ne soient pas dans de mauvaises conditions, qu'ils n'aient pas froid, qu'ils n'aient pas faim »

Le constituant thématisé peut précéder les mots introduisant les phrases interrogatives, les souhaits et les injonctions.

- l'antéposition par rapport au sujet :

(7) Hac uirtute maiores uestri primum uniuersam Italiam devicerunt (Cic. Phil. 4,5)

" C'est par ce courage que vos ancêtres sont d'abord parvenus à vaincre toute l'Italie » 
Il est difficile d'établir si une pause intervient après le Thème antéposé. Les éditeurs n'introduisent généralement pas de ponctuation, ni pour les exemples de (6), ni pour le type illustré par (7). Il n'est pas pour autant exclu que (6) et (7) aient des intonations différentes.

On voit en tout cas que l'extraposition correspond à deux effets interprétatifs différents : la Thématisation (6) et la Focalisation (7)) ; la question est alors de savoir si le latin présente des critères syntaxiques permettant de les distinguer.

1.2. On sait que le français distingue deux formes de déplacement à gauche : a) la Thématisation par dislocation gauche, avec reprise anaphorique :

(8) Pierre, on va lui parler

Le constituant disloqué n'est pas directement gouverné par le verbe; il est repris par un anaphorique qui présente la forme requise par sa fonction dans la phrase.

b) la Focalisation par antéposition, sans reprise anaphorique :

(9) A Pierre j'ai parlé de son travail (et à Paul de ses vacances)

Le constituant déplacé conserve la forme correspondant à sa fonction.

Il est tentant d'appliquer le même type de distinction au latin. En effet, si on compare (1)-(3) d'une part et (4) d'autre part, on observe une distribution morphologique comparable au français. Lorsque le constituant antéposé présente une forme qui ne lui confère pas de fonction dans la proposition, il est repris par un pronom anaphorique. Cette reprise peut s'effectuer à l'aide d'un pronom plein (is en (1a), ibi en (3)) ou bien par un pronom nul (sujet de essent en (1b) ou de est en (3b)).

En revanche, lorsque le syntagme antéposé présente une forme qui le rend directement interprétable dans la proposition, comme en (1c) ou (4) par exemple, il n'est pas repris par un anaphorique. Mais, contrairement au français, cette combinaison ne sert pas spécifiquement à la Focalisation du constituant: si (1c) présente une Focalisation contrastive du Thème, (4) relève simplement de la Thématisation.

On peut résumer les observations précédentes à l'aide du tableau suivant :

\begin{tabular}{|c|c|c|}
\hline$(10)$ & Thématisation & Focalisation \\
\hline $\begin{array}{c}\text { Dislocation : } \\
- \text { nominativus pendens }\end{array}$ & oui & non \\
$-d e+$ ablatif & & \\
a avec reprise & oui & non \\
a sans reprise & oui & oui \\
\hline Antéposition & oui & oui \\
\hline
\end{tabular}


1.3. La bipartition précédente entre dislocation et antéposition se heurte en outre aux difficultés suivantes :

a) L'existence de pronoms nuls - admise ici, suivant les théories aussi bien chomskyenne que fonctionaliste - rend difficile l'observation du contraste précédent : on pourrait, par exemple, soutenir qu'il n'y a pas de reprise anaphorique en (1b) ou inversement qu'il y a un pronom nul en (6).

b) On admet que les exemples de (4) relèvent d'un déplacement à gauche sur l'observation quasi exclusive de l'ordre des mots, en supposant que le placement initial d'un constituant joue le même rôle dans une langue à ordre libre, comme le latin, et dans des langues à ordre fixe, comme l'anglais ou le français. Or on sait que des paramètres très variés et hétérogènes entrent en ligne de compte dans l'organisation syntagmatique (cf. Rosén 1992, Cabrillana 1996).

A la place dans la chaine syntagmatique s'ajoutent des critères formels : quand le Thème est un $\mathrm{SN}$ déjà identifié, il est accompagné de déterminants de type démonstratif-anaphorique, comme en (4a). Mais ces déterminants ne sont pas spécifiques de la Thématisation. En revanche le relatif de liaison, illustré par (4b), est doublement caractéristique de la Thématisation, et par sa forme de relatif et par sa position initiale.

c) Les données elles-mêmes conduisent à renoncer au contraste précédent entre dislocation et antéposition : la reprise anaphorique s'observe aussi pour un SN dont la forme permettrait de l'interpréter directement dans la proposition :

(11a) Amicos domini, eos babeat sibi amicos (Cato agr. 5,3)

«Les amis du maitre, qu'il les considère comme ses amis à lui »

(11b) signa quae nobis curasti, ea sunt ad Caietam exposita. (Cic., Att.1,3,2)

«Les statues que tu m’as procurées, elles ont été débarquées à Caï̀te. »

(11c) nostro seni buic stolido, ei profecto nomen facio ego Ilio (Plaut. Bacch. 945)

«Notre imbécile de vieillard que voici, je lui donne, c'est garanti, le nom d'Ilion»

mais dans ce cas, seule l'interprétation comme Thème est permise.

Nous pouvons alors raffiner le tableau de (10):

\begin{tabular}{|l|l|l|}
\hline & Thématisation & Focalisation \\
\hline Antéposition avec reprise & oui & non \\
\hline Antéposition sans reprise & oui & oui \\
\hline
\end{tabular}

Reste le point soulevé sous a) : doit-on considérer que les exemples de (4)-(6) et (11) présentent la même strucuture, avec un pronom anaphorique tantôt vide (4)-(6), tantôt plein (11), ou bien qu'ils sont distincts et donc que le terme antéposé n’occupe pas la même position syntaxique? Nous reviendrons sur cette question dans la dernière section. 


\section{Thématisation dans les propositions subordonnées}

Dans la phrase complexe, la Thématisation peut concerner aussi bien la proposition enchâssante que la proposition enchâssée. La question se pose donc de savoir si ce sont les mêmes procédés qui sont mis en jeu dans les deux types de proposition et si la Thématisation peut concerner simultanément les deux propositions.

\subsection{Propositions temporelles et conditionnelles}

Parmi les exemples de (6) illustrant la Thématisation par antéposition, (6b) présente la Thématisation d'un constituant d'une proposition enchâssée. L'antéposition se double d'un franchissement du subordonnant marquant le début de la proposition enchâssée.

Ce phénomène $\mathrm{a}$, semble-t-il, une origine historique : les subordonnants auraient anciennement occupé la position seconde de la proposition; par la suite, avec le développement des subordonnants comme démarcateurs de proposition, les termes précédant le subordonnant auraient reçu une interprétation spécifique. Cette sortie d'un constituant au-delà des limites du domaine propositionnel a reçu le nom de traiectio ("traversée", traduction du grec 'hyperbate'). C'est d'ailleurs en termes de Transformation par Montée que la Grammaire Générative a rendu compte du phénomène.

En latin classique, les propositions circonstancielles régulièrement antéposées connaissent la traiectio et de ce fait on trouve en tête de la phrase un constituant Thématisé :

a) avec les conditionnelles :

(13a) Romani si casu interuenerint ... (Caes. Gall. 7,20,4)

«Si les Romains étaient intervenus par hasard...»

(13b) Haec si grania aut acerba nideantur ... (Caes. Gall. 7,14,10)

« Si ces mesures paraissent cruelles ou dures ...»

La littérature didactique rend explicite la parenté entre de + ablatif et l'antéposition par traiectio:

(14) De uinea uetere. Vineam ueterem si in alium locum transferre uoles... (Cato agr. 49,1) «Concernant une vieille vigne. Une vieille vigne, si on veut la transplanter ailleurs...»

b) avec les temporelles :

L'enchaînement thématique conduit souvent dans les textes narratifs à l'antéposition d'une temporelle définissant le cadre dans lequel se place l'événement nouveau; le constituant servant de transition et appartenant à cette temporelle est lui-même anticipé :

(15a) Eo cum nenisset, magna difficultate adficiebatur... (Caes. Gall. 7,6,2)

"Une fois arrivé là, il était aux prises avec une grande difficulté »

(15b) Quin etiam Caesar cum in opere singulas legiones appellaret... (Caes. Gall. 7,17,4) «Bien plus comme César visitant les travaux adressait la parole à chaque légion ...» 


\subsection{Les propositions complétives conjonctives}

Pour les propositions complétives, nous retrouvons deux procédés de Thématisation déjà répertoriés : l'antéposition d'un constituant de la forme de + ablatif et la traiectio:

(16a) de fratre ubi eum nisuri essemus nesciebamus (Cic. Att. 3,7,3)

« concernant mon frère, j’ignorais où nous le verrions »

(16b) ornamenta quae locaui metuo ut possim recipere (Plaut. Curc. 464)

« les ornements que j’ai laissés en gage, je me demande si je pourrai les récupérer »

Ces deux constructions sont concurrencées par un tour un peu différent, l'accusatif proleptique :

(17a) bunc comparem metuo meum / ne deserat med (Plaut. Pseud. 1026)

«mon compagnon que voici, j'ai peur qu'il ne m'abandonne»

(17b) Nam sanguinem, bilem, pituitam, ossa, .. uideor posse dicere unde concreta et quo modo facta $\operatorname{sint}$ (Cic. Tusc.1,56)

«En effet le sang, la bile, la pituite, les os, je crois pouvoir dire de quels éléments ils sont constitués et de quelle manière ils sont produits »

Dans cette dernière construction, le constituant apparemment "sorti" de la proposition subordonnée prend la forme d'un complément d'objet du verbe principal.

Deux indices confirment la grande parenté entre traiectio et accusatif proleptique :

- la symétrie observée entre les deux constructions :

(18) Metuo fratremaCC. / ne intus sit; porro autem paterNom. ne rure redierit (Ter. Eun. 610611) «J'ai peur que mon frère ne soit à l'intérieur; et en plus que mon père ne soit revenu de la campagne »

- les variations dans la tradition manuscrite :

(19) qui rogo pallam $\mathrm{ACC}_{\text {. }}\left(\right.$ manuscrit $\mathrm{D}^{2}$ ) / palla $\mathrm{NOM}_{\mathrm{N}}$ ut referatur rursum ad uxorem meam (Plaut. Men. 907)

«moi qui demande que le manteau soit rapporté à ma femme»

Certains exemples sont même parfaitement ambigus :

(20) fores facite ut pateant (Plaut. Stich. 309)

«Faites que les portes soient ouvertes»

Fores peut morphologiquement être aussi bien un nominatif qu'un accusatif, et donc relever de la traiectio aussi bien que de la prolepse.

Il existe cependant des différences entre les deux constructions :

a) seul un SN peut donner lieu à prolepse, tandis que n'importe quel constituant peut être extraposé par traiectio: 
(21) rogare iussit ted ... / mecum simitu ut ires ad sese domum. (Plaut. Stich. 248-249) « Il m'a ordonné de te demander d'aller en même temps que moi chez lui »

b) un seul SN à la fois peut donner lieu à prolepse, tandis que plusieurs constituants peuvent être simultanément extraits par traiectio:

(22) quanto, nox, fuisti longior hac proxuma, / tanto breuior dies ut fiat faciam (Plaut. Amph. 548-549)

« nuit, d'autant tu as été plus longue que la nuit précédente, d'autant je ferai que soit plus court le jour»

c) seul le $\mathrm{SN}$ sujet de la proposition subordonnée peut donner lieu à la prolepse (cf. 18 et 19);

d) un accusatif proleptique peut apparaitre à droite de la subordonnée (dislocation droite), alors que la traiectio à droite n'est pas formellement identifiable :

(23) nisi scio quo agas te (Plaut. Pers. 234)

«si je ne sais où tu vas, toi »

En fait, la concurrence entre les deux constructions n'est qu'apparente.

En premier lieu, le phénomène de traiectio est si usuel (cf. la fréquence des exemples comme (13)) et si peu contraint (pratiquement tous les constituants d'une subordonnée peuvent être extraits simultanément) qu’on peut douter, avec Marouzeau (1953), qu'il y ait à chaque fois un relief informatif particulier.

Même en admettant que la traiectio traduise une mise en relief de l'information, il n'y a pas de distribution complémentaire: traiectio et accusatif proleptique servent également à la Thématisation et à la Focalisation. Nous avons vu en effet que le constituant extrait par traiectio était Thématisé en (16b) et Focalisé en (21); de même l'accusatif proleptique constitue le Thème en (17) et le Focus dans l'exemple suivant:

(24) quoniam tu me et ego te qualis sis scio (Plaut. Aul. 217)

« puisque toi, tu sais qui je suis, moi et que moi, je sais qui tu es, toi »

\subsection{La proposition infinitive}

Parmi les propositions complétives, la proposition infinitive est caractérisée par l'absence de subordonnant. Il s'ensuit que ce type de proposition ne connait pas de démarcation explicite. On peut alors se demander si la Thématisation d'un constituant est visible en l'absence de critère intonatif.

Nous proposerons de reprendre le critère positionnel observé pour les autres types de propositions : la place initiale peut être l'indice d'une Thématisation pour peu que le SN initial soit perçu comme extrait de la proposition infinitive. La dislocation est rendue perceptible par différents moyens annexes :

a) l'insertion d'un adverbe, apostrophe ou incise qui détache ce $\mathrm{SN}$ de sa proposition : 
(25) Auditisne magian, qui eam temere accusatis, artem esse dis immortalibus acceptam ...? (Apul. apol. 26,1)

«Entendez-vous que la magie, vous qui l'accusez imprudemment, est un art agréable aux dieux?»

b) l'antéposition par rapport au verbe introducteur

Le phénomène est attesté dès le latin archaïque, y compris dans les inscriptions :

(26a) HONC OINO / PLOIRVME COSENTIONT ... OPTVMO FVISE VIRO $\left(\mathrm{CIL} \mathrm{I} \mathrm{I}^{2} / 2,9,1-2\right)$ «Il est le seul dont on pense généralement qu'il a été un homme parfait »

(26b) Haec ego non possum dicere non esse hominis quamuis et belli et humani (Cic. fin. 2,102) «Ces paroles, je ne peux pas nier qu'elles sont d'un homme aussi aimable et bon qu'on voudra »

Mais comme pour toutes les antépositions par traiectio, l'interprétation Focus est attestée dans les mêmes conditions :

(27) "aio te, Aeacida, Romanos vincere posse" (Ennius apud Cic., div. 2,56)

"J'affirme que toi, Eacide, tu peux vaincre les Romains / que toi, Eacide, les Romains peuvent te vaincre»

\section{Thématisation et structure syntaxique}

Nous avons vu à partir des exemples (12) que les constituants thématisés étaient en relation de coréférence avec un autre constituant appartenant à un domaine syntaxique qui permet cette mise en relation.

Pour rendre compte des différents types de Thématisation décrits plus haut, nous pouvons les ramener dans un premier temps à une structure commune :

(28) $\left[\mathrm{x}\right.$ Thèmè $\left.\mathrm{i}\left[\mathrm{Y} \ldots \mathrm{e}_{\mathrm{i}} \ldots\right]\right]$

dans laquelle $\mathrm{X}$ désigne la catégorie qui domine à la fois le Thème et la catégorie de reprise, Y la catégorie qui domine la catégorie de reprise et $e$, la reprise.

Il importera ensuite d'identifier ces catégories.

\subsection{Identification de e}

Si on suit le modèle chomskyen du Gouvernement et du Liage, la catégorie de reprise peut être identifiée avec

a) une trace de mouvement syntaxique

b) une anaphore, nulle ou pleine 
L'identification de la relation anaphorique ne pose pas de problème quand la reprise est représentée par un pronom plein, comme en (1a) pour de + abl. ou en (3) pour le nominativus pendens. On peut en déduire que (1b) présente également une anaphore, mais sous la forme d'un pronom nul.

Les syntagmes extraposés, comme le nominativus pendens et de + abl., constituent les interprétants prioritaires du pronom. Ils ne fonctionnent pas pour autant comme les lieurs d'une anaphore liée : le réfléchi de $3^{e}$ personne ne se rencontre pas, alors qu'il apparait dès lors que l'anaphore renvoie au $\mathrm{SN}$ le plus haut dans le domaine syntaxique (cf. Milner 1982).

Dans le cas de la traiectio, la reprise anaphorique ne s'observe pas, ce qui conduit à parler d'un déplacement du SN hors de son domaine phrastique. D'un point de vue technique, l'interprétation se fait alors en mettant en relation ce syntagme extraposé avec la trace qu'il laisse à l'intérieur de la proposition. L'interprétation du SN extraposé est double : par sa position, il est interprété comme Thème de l'ensemble de l'énoncé et par sa forme, il est rapporté à sa fonction interprétative à l'intérieur de la proposition.

Le cas de l'accusatif proleptique est particulièrement intéressant: par sa position et par sa forme, il devrait être interprété à l'intérieur de la proposition où il se trouve ; or ce n'est pas le cas. La raison en est double :

- malgré sa forme d'accusatif, il n'est pas correctement interprétable comme un argument du verbe principal ;

-il est interprété comme le sujet de la proposition enchâssée ;

A la différence de la traiectio, la reprise anaphorique pleine est possible :

celabat metuebatque te, ne tu sibi persuaderes... (Plaut. Truc. 201)

«elle te le cachait; elle avait peur que tu ne voulusses la contraindre... »

Il n'y a donc pas lieu de traiter l'accusatif proleptique en termes de déplacement, mais plutôt de relation anaphorique entre le $\mathrm{SN}$ Thème et un pronom de reprise, le plus souvent nul (cf. Milner 1980, Touratier 1978, Maraldi 1986, Bortolussi 1998). Tout se passe en fait comme si une phrase à nominativus pendens devenait subordonnée : le nominatif n'est plus possible, parce que la phrase n'est plus embrayée directement ; il est remplacé par l'accusatif qui est son équivalent pour les phrases nonembrayées (cf. Milner 1982 à propos de l'AcI).

La relation entre le $\mathrm{SN}$ prolepsé et sa reprise anaphorique est contrainte, à la manière d'une anaphore liée : seul le sujet de l'enchâssée peut être coréférentiel. Il y a une raison interprétative : le sujet de la proposition constitue le Topique de la phrase dans ses formes non-marquées; il a donc une sorte de priorité pour devenir le Thème de l'ensemble de la proposition; d'un point de vue syntaxique, il existe une contrainte, dite du Sujet-spécifié qui bloque la mise en relation d'une anaphore avec un antécédent quand la proposition contient un sujet au nominatif.

\subsection{Identification de la position occupée par le Thème}

Depuis les travaux de Rizzi (1997) sur la périphérie gauche de la phrase, on admet dans la Théorie dite Minimaliste de Chomsky qu'existe à gauche de la forme propositionnelle (IP) une série de catégories fonctionnelles : 
(30) (Force Top(ique1) Foc(us) Top(ique2) Fin(itude)) IP l'énoncé.

Il existe donc deux positions Top(ique) susceptibles d'accueillir le Thème de

Nous ferons l'hypothèse que le Thème occupe en latin une position externe au domaine propositionnel, qu'il soit ou non déplacé depuis une position interne à la phrase. Les conséquences sont multiples :

- la position TOP peut être remplie par des constituants de formes très variées ; si l'interprétation comme Thème provient de leur position syntaxique, c'est leur forme qui déterminera leur mode d'intégration syntaxique à la proposition ;

- si la position TOP est du même type que la position COMP, on s'attend à observer des phénomènes comparables à ceux qui ont été décrits pour les mots QU- ;

- une phrase complexe peut contenir a priori autant de positions TOP qu'il y a de propositions. La concurrence entre les différents types de Thématisation se pose alors autrement : peuvent-ils se combiner? et comment?

3.2.1. La forme prise par le Thème conduit à différents types d'intégration syntaxique à la proposition qui constitue l'information rhématique :

a) Le nominatif et le syntagme de + abl. ont ceci de commun qu'ils permettent d'employer un SN 'hors syntaxe'. Cette caractéristique se retrouve dans leur emploi comme mention ou titre :

(31) Laelius de amicitia, Cato de senectute (traités de Cicéron intitulés Lélius ; l'amitie et Caton; la vieillesse)

Les deux formes ne sont pourtant pas équivalentes d'un point de vue syntaxique. Le syntagme $d e+$ abl. entre dans la série des compléments circonstanciels : il s'agit de SP adjoints au SV. Ce constituant est polyvalent: outre ses emplois temporel et local, il indique le domaine d'application ou d'extension de la prédication (de senectute librum scribere: écrire un livre sur la vieillesse) ou de l'énoncé lui-même. Le partage entre ces variantes est délicat : en (1b) de Hispaniis peut s'analyser simplement comme un complément circonstanciel dépendant de dubitabant ou bien comme un complément de phrase.

Dans la logique de Milner (1982, p. 275-280), le nominatif est la marque casuelle assignée à un SN directement embrayé, que l'embrayage soit extérieur au domaine propositionnel, ou qu'il soit interne, sous la forme du Spécifieur de IP.

b) Les propositions du type quod + indicatif ont ceci de remarquable qu'elles ont la forme d'une subordonnée - ce qui suppose leur intégration syntaxique à une autre proposition - mais elles ne remplissent pas de fonction dans cette proposition. On pourrait soutenir la même chose concernant la proposition relative antéposée de (2b).

c) L'accusatif proleptique présente la forme d'un complément d'objet du verbe enchâssant, sans remplir le rôle sémantique spécifique de ce verbe. Il ressemble sur ce point à $d e+$ abl. avec lequel il est en concurrence dans la phrase complexe. 
d) L'antéposition avec reprise anaphorique illustrée par (11) se présente comme une alternative au nominativus pendens: le SN antéposé est pareillement non-gouverné ; mais cette fois il reçoit sa marque casuelle de l'anaphorique, par un accord "inverse". Remarquons que ce phénomène n'est pas isolé en latin, puisque l'accord de l'antécédent avec le pronom anaphorique s'observe également quand une proposition relative est antéposée :

(32) Sed istum quem quaeris ego sum (Plaut. Curc. 419)

"Quant à l'homme que tu cherches, c'est moi. »

Pour compléter le parallélisme, l'ensemble antécédent + relative peut se confondre avec le nominativus pendens dans un exemple comme :

(33) Muliernom. quae se suamque aetatem spernit, speculo ei DAT. usus est (Plaut. Most. 250) «La femme qui n'est pas sûre d'elle et qui craint de vieillir, c'est elle qui a besoin d'un miroir»

e) l'antéposition sans reprise anaphorique semble présenter une intégration totale à la proposition : la forme qu'elle présente permet l'identification de sa fonction dans la proposition. Seule en fait sa place dans la chaîne syntagmatique attire l'attention. Ceci n'impose pas a priori de recourir à une explication syntaxique; les alternatives sont connues : simple déplacement superficiel "stylistique" ou informationnel.

Si nous retenons l'explication syntaxique, nous pouvons rapprocher la "montée" des constituants Thématisés de la montée des quantificateurs : le SN monté en position TOP est gouverné par sa trace et reçoit d'elle la marque casuelle. Aucune contrainte particulière ne pèse sur ce mouvement, c'est pourquoi n'importe quel constituant peut être déplacé en position TOP.

3.2.2. Avant la proposition de Rizzi concernant la périphérie gauche de la phrase, celle-ci était occupée par la catégorie fonctionnelle COMP. On a remarqué depuis longtemps que, dans la phrase complexe, les termes QU- pouvait remonter de position COMP en position COMP :

(34a) L'bomme que je crois que tu as vu

(34b) L'bomme i [COMP que $e_{i}$ [IP je crois [COMP que [IP tu as vu $\left.\left.\left.t_{i}\right]\right]\right]$

Le pronom relatif que, complément de as $v u$, a été déplacé successivement en position COMP de la complétive, puis de la relative. Comme l'observe Touratier (1994, p. 600), l'accusatif proleptique, en tant que Thème, peut également remonter en position COMP pour former une relative :

(35) aliqua culpa, [comp quam, tu wereris [comp ne proi a te suscepta videatur]] (Cic. Planc. 52) quelque faute que tu crains que par toi commise semble « quelque faute dont tu crains qu'elle ne semble avoir été commise par toi »

Il est possible d'étendre ce principe aux positions spécifiques de Thème. Les constituants en de + abl. illustrent ce phénomène, puisqu'on les trouve systémati- 
quement remontés en tête de la phrase complexe (cf. (1)). Concernant les accusatifs proleptiques, si on compare les phrases suivantes:

(36a) Dic modo bominem qui sit (Plaut. Bacch. 555)

«Dis moi seulement, cet homme, c'est qui ?»

(36b) Viniam sic facito uti curetur (Cato agr. 33,1)

«La vigne, voici comment elle doit être soignée »

il apparait qu'en (36a) bominem constitue le Thème sur lequel porte la demande d'information, tandis qu'en (36b) viniam est le Thème de l'ensemble de la phrase, et pas seulement de la subordonnée.

On peut proposer la généralisation suivante :

-lorsque la Thématisation concerne seulement la subordonnée, l'accusatif proleptique est immédiatement à gauche de la proposition subordonnée :

(36a') [IP Dic modo [TOP hominemi [c qui [IP proi sit]]]]

-lorsque la Thématisation concerne l'ensemble de la phrase complexe, l'accusatif proleptique est déplacé en tête de phrase, avec une dislocation de l'argument complexe Thème+Subordonnée par-dessus le verbe recteur :

(36b') [тор Viniami [IP sic facito [c uti [IP proi curetur]]]]

La même logique prévaudra dans l'analyse de la traiectio, quand celle-ci correspond à une Thématisation :

(37) ornamenta quae locaui metuo ut possim recipere (Plaut. Curc. 464)

« les ornements que j’ai laissés en gage, je me demande si je pourrai les récupérer»

Dans le même contexte didactique qu'en (36b), on retrouve en effet la structure précédente :

(38) Aratra nomeresque facito uti bonos babeas (Cato agr. 5,6)

"Concernant les araires et les socs, veille à en avoir de bons »

Pour ce qui concerne les propositions infinitives, seul le déplacement en tête de phrase, avec une dislocation par-dessus le verbe recteur permettra d'identifier la Thématisation. Il faudrait disposer d'autres critères que la position pour identifier les Thématisations à gauche de la subordonnée.

\subsubsection{Combinaison de prolepse et traiectio}

On peut trouver simultanément un accusatif proleptique et une traiectio :

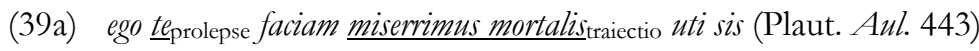

«Toi, je vais faire en sorte que tu sois le plus malheureux des mortels »

(39b) Sosia $\underline{m e}_{\text {prolepse }}$ ille egomet egraectio fecit sibi $_{\text {traiectio }}$ ut crederem (Plaut. Amph. 598)

«Moi, ce Sosie a fait en sorte que moi-même je le crois lui » 
L'accusatif proleptique joue le rôle de Thème de la prédication enchâssée, tandis que les constituants extraits par traiectio ont l'interprétation Focus. Il ne semble pas que la structure inverse existe. Il est plus difficile en revanche de déterminer si les deux peuvent être simultanément supports d'une Thématisation. On sait en revanche que la double Thématisation existe dans la combinaison d'une traiectio et de de + abl. :

(40) Quid? idem iste, inquam, de noluptate quid sentit? (Cic. fin.2,8)

«Eh quoi? ce même homme, dis-je, au sujet du plaisir, quelle est son opinion?»

Idem iste est la reprise du Thème dans une progression thématique, tandis que de uoluptate constitue le nouveau Thème secondaire. En revanche la combinaison de l'accusatif proleptique et de de + abl. ne semble pas attestée.

\section{Conclusion}

Les phénomènes de Thématisation observés en latin sont très proches de ceux que l'on observe dans les langues-filles : syntagme prépositionnel en tête et dislocation gauche. L'originalité du latin réside davantage dans la coexistence de constructions concurrentes, comme la traiectio et l'accusatif proleptique, lequel a un équivalent français :

(41a) devine quelle personne j'ai rencontrée

(41b) devine la personne que j'ai rencontrée

Les problèmes de Thématisation soulèvent une question beaucoup plus vaste pour le latin : la relation entre l'organisation syntagmatique observable et la structure syntaxique. On sait que le latin passe pour une langue à ordre libre; or les phénomènes de Thématisation sont crucialement liés à certaines places, et de plus ces places sont les mêmes que dans les langues à ordre fixe. Une hypothèse est que ces phénomènes de placement relèvent de la syntaxe et pas seulement de l'organisation informative. Les différences de fonctionnement entre prolepse et traiectio, leur combinaison, les contraintes syntaxiques pesant sur la prolepse, la remontée du Thème dans les phrases complexes sont autant d'arguments en faveur d'une analyse syntaxique de la Thématisation en latin. 


\section{REFERENCES}

AMACKER R., 2001, "Indices de thématisation segmentée non initiale chez Cicéron » in C. Moussy (éd.) De lingua latina novae quaestiones, Louvain - Paris, Peeters, p. 185-199.

AMACKeR R., 1998, «Ordre des mots et subordination : la traiectio chez Varron » in B. GarciaHernandez (éd.) Estudios de Linguïstica Latina, Actas del IX Coloquio Internacional de Linguïstica Latina, Madrid, Ediciones Clasicas, p. 139-154.

Bodelot C., 1996, «Propositions complétives détachées en latin: dislocation gauche vs dislocation droite ", in A. Bammesberg, F. Herberlein (éds.) Akten des VIII. internationalen Kolloquium zur lateinischen Linguistik, C. Winter, Heidelberg, p. 233-248.

BOLKESTEIN A.-M., 1981, «Embedded predications, displacement and pseudo-argument formation in Latin» in A.-M. Bolkestein et alii (éds.) Predication and Expression in Functional Grammar, London, Academic Press, p. 63-112.

BorTOLussi B., 1998, «Facite uentum ut gaudeam. Quelques phénomènes d'ambiguïté syntaxique » in B. Garcia-Hernandez (éd.) Estudios de Linguïstica Latina, Actas del IX Coloquio Internacional de Linguïstica Latina, Madrid, Ediciones Clasicas, p. 203-216.

Cabrillana C., 1996, "Multifunctional analysis of word order », in H. Rosén (éd.) Aspects of Latin. Papers from the 7 th international colloquium on Latin linguistics, Jerusalem, Innsbruck, p. 377-388.

CHOMSKY N., 1981, Lectures on Government and Binding, Dordrecht, Foris.

Christol A., 1989, « Prolepse et syntaxe indo-européenne » in G. Calboli (éd.) Subordination and others Topics in Latin, Amsterdam, Benjamins, p. 65-89.

LONGREE D, 1996, «'Relatives en rallonge' ou 'relatif de liaison' : l'exemple de Tacite », in A. Bammesberg, F. Herberlein (éds.) Akten des VIII. internationalen Kolloquium zur lateinischen Linguistik, C. Winter, Heidelberg, 268-281.

Maraldi M., 1986, «Proleptic accusative » in G. Calboli (éd.) Papers on Grammar II, Bologne, CLUEB, p. 87-105.

Marouzeau J., $\quad 1949$, L'ordre des mots dans la phrase latine, Paris, Belles Lettres.

1953, L'ordre des mots en latin. Volume complémentaire, Paris, Belles Lettres.

MiLNER J.-C., 1980, « La prolepse en grec ancien », Lalies I, p. 39-52.

MiLner J.-C, 1982, Ordres et raisons de langue, Paris, Seuil.

PINKSTER H., 1984, Latinjse syntaxis en semantiek, Amsterdam, Grüner (trad. angl. 1988, Tübingen, Francke).

RIZZI L, 1997, "The fine structure of the left-periphery », in L. Haegeman (éd.) Elements of Grammar: Handbook of Generative Syntax, Dordrecht, Kluwer, p. 281-337.

RosÉN H., 1996, «Die Arten der Prolepse im Lateinischen in typologischer Sicht», in C. Kroon, R. Risselada (éds.) On Latin, Linguistic and Literary Studies in honour of Harm Pinkster, Gieben, Amsterdam, p. 244-262.

TOURATIER Chr., 1980, « L'accusatif proleptique en latin », Lalies I, p. 53-56.

TOURATIER Chr., 1994, Syntaxe latine, Paris-Louvain, Peeters. 
